\title{
Signal Denoising Method Based on Adaptive Redundant Second-Generation Wavelet for Rotating Machinery Fault Diagnosis
}

\author{
Na Lu, ${ }^{1}$ Guangtao Zhang, ${ }^{2}$ Yuanchu Cheng, ${ }^{3}$ and Diyi Chen ${ }^{4}$ \\ ${ }^{1}$ School of Water Conservancy \& Environment, Zhengzhou University, Zhengzhou 450000, China \\ ${ }^{2}$ Henan Electric Power Research Institute, Zhengzhou 450000, China \\ ${ }^{3}$ School of Power and Mechanical Engineering, Wuhan University, Wuhan 430072, China \\ ${ }^{4}$ College of Water Resources and Architectural Engineering, Northwest A\&F University, Yangling 712100, China
}

Correspondence should be addressed to Yuanchu Cheng; yccheng@whu.edu.cn

Received 17 January 2016; Revised 9 August 2016; Accepted 10 August 2016

Academic Editor: Stefan Balint

Copyright (C) $2016 \mathrm{Na}$ Lu et al. This is an open access article distributed under the Creative Commons Attribution License, which permits unrestricted use, distribution, and reproduction in any medium, provided the original work is properly cited.

\begin{abstract}
Vibration signal of rotating machinery is often submerged in a large amount of noise, leading to the decrease of fault diagnosis accuracy. In order to improve the denoising effect of the vibration signal, an adaptive redundant second-generation wavelet (ARSGW) denoising method is proposed. In this method, a new index for denoising result evaluation (IDRE) is constructed first. Then, the maximum value of IDRE and the genetic algorithm are taken as the optimization objective and the optimization algorithm, respectively, to search for the optimal parameters of the ARSGW. The obtained optimal redundant second-generation wavelet (RSGW) is used for vibration signal denoising. After that, features are extracted from the denoised signal and then input into the support vector machine method for fault recognition. The application result indicates that the proposed ARSGW denoising method can effectively improve the accuracy of rotating machinery fault diagnosis.
\end{abstract}

\section{Introduction}

At present, rotating machinery has been widely applied in industrial field. Meanwhile, its safety and stability operating problems have gained comprehensive attention $[1,2]$. Rotating machinery failure often causes equipment damage, significant economic losses, and even causalities. Therefore, its fault diagnosis is of great significance for ensuring the safety and stability operation as well as the prevention of catastrophe accidents. Vibration signal processing and analysis are one of the most favored means to diagnose the rotating machinery fault $[3,4]$. However, because of the complicated operating conditions and harsh operating environment, the vibration signal is often submerged in a large amount of noise, which may decrease the accuracy of the fault diagnosis [5-7]. Therefore, it is necessary to study an effective vibration signal denoising method to improve the accuracy of the rotating machinery fault diagnosis.
Signal denoising problem has always been the hotspot in signal processing field. Up to now, various signal denoising methods have been developed to analyze the vibration signals, such as finite impulse response filter (FIR) [8], time-frequency manifold [9], empirical mode decomposition (EMD) [10], curvelet transform [11], quantum Hadamard transformation [12], wavelet [13], and multiwavelets [14]. Among these methods, FIR and wavelet are two of the most popular time-frequency analysis tools. However, the frequency response of FIR depends on its coefficients, which is related to the cut-off frequency and the order of the filter [15]. Hence, the choice of cut-off frequency and order may have a great impact on the denoising result. Wavelet has been widely applied for mechanical vibration signal denoising [1618]. However, the denoising effect is influenced by the wavelet base function selection to a large extent [19]. Inappropriate base function may lead to unsatisfactory denoising result. Furthermore, the existing wavelet base functions cannot 
change adaptively according to the characteristics of the acquired vibration signal. This may degrade the denoising result [20].

Second-generation wavelet (SGW) is a new wavelet theory that emerged in recent years. Compared with the traditional wavelet, the construction of SGW avoids Fourier transform. In addition, it has fast computational speed [21]. Because of the advantages of SGW, it has been widely applied in vibration signal processing field. For example, Li et al. [22, 23] combined lifting wavelet with morphological wavelet to construct an adaptive morphological gradient lifting wavelet, which is used for bearing and gear vibration signal denoising and feature extraction; Bao et al. [24] adopted lifting wavelet for weak fault feature extraction from vibration signal; Fan et al. [25] applied SGW to decompose bearing vibration signal and then input the result to independent component analysis (ICA) approach for fault diagnosis; Chen et al. [26] employed SGW to test and diagnose crack location of rotor; Bao et al. [27] applied two-dimensional lifting wavelet for rotating mechanical vibration data compression, and so forth. Even though multiple achievements have been obtained, SGW suffers from some defects. Because the split and merge operations are needed in the SGW transformation process, frequency aliasing problem often emerged. This makes the decomposition result contain fake frequency components and decreases the fault diagnosis accuracy.

To overcome the frequency aliasing problem, [24] analyzed the reason why it appears and proposed a redundant second-generation wavelet (RSGW) method. RSGW transform does not include split and merge operations and thus can overcome the frequency aliasing problem to a large extent. Therefore, RSGW is superior to SGW in application to signal denoising. However, similar to the base function of wavelet, the prediction operator length, update operator length, and decomposition level of RSGW should be predetermined. In general, these parameters have a great impact on signal denoising performance. But it is difficult to select them properly.

In order to select the optimal parameters of RSGW according to the characteristics of signal and obtain a favorable denoising result, an adaptive redundant secondgeneration wavelet (ARSGW) denoising method for rotating machinery fault diagnosis is proposed in this paper. This method is mainly composed of three phases. In the first phase, parameters like the prediction operator length, update operator length, and decomposition level of RSGW are initialized to some default values within their certain range. In the second phase, the optimal value of an index for signal denoising result evaluation and the genetic algorithm are taken as the optimization objective and the optimization algorithm, respectively, to search for the optimal values of these parameters. After that, the RSGW with optimal parameters is applied to vibration signal denoising for rotating machinery in the last phase. It can be seen that an effective index for signal denoising result evaluation is essential for optimal parameters searching in the second phase of this method. Signal-to-noise ratio (SNR) [28] is a commonly used index for evaluation of noise intensity in signal. However, it can only be used in the circumstance that the real signal without noise component is known beforehand. Because the real signal component in the actual vibration signal is unavailable, SNR is not applicable for its denoising result evaluation. Therefore, a new index for rotating machinery signal denoising result evaluation (IDRE) is constructed in this paper. Based on IDRE, the newly proposed method can enable the parameters of RSGW to change adaptively with the characteristics of the acquired vibration signal and improve the signal denoising performance effectively.

The rest of this paper is arranged as follows. In Section 2, theory of SGW and RSGW is reviewed briefly. In Section 3, a new IDRE is constructed and the ARSGW denoising method is proposed. Then this method is applied for a hydroturbine unit vibration simulation signal denoising in Section 4 to demonstrate the effectiveness of the IDRE and the ability of ASGW to obtain an optimal denoising result. In Section 5, vibration signals of a rotating machinery system are denoised. Features in both time and frequency domains are extracted from the denoised signals and then input into the support vector machine method for fault recognition to validate the ability of ARSGW denoising method to improve the accuracy of rotating machinery fault diagnosis. Conclusions are given in Section 6.

\section{Redundant Second-Generation Wavelet}

2.1. Summary of Second-Generation Wavelet Theory. As a branch of wavelet theory, SGW inherited its excellent characteristics of time-frequency location. Furthermore, SGW has higher calculation efficiency and more clear principle and needs lower space [24]. SGW transform includes decomposition and reconstruction processes demonstrated in Figure 1. The decomposition process of SGW includes the following steps:

(1) Split: the signal $X=\{x[n], n \in Z\}$ is divided into two subsets: the odd sample set $X_{o}=\left\{x_{o}[n], n \in Z\right\}$ and the even sample set $X_{e}=\left\{x_{e}[n], n \in Z\right\}$ :

$$
\begin{aligned}
& x_{o}[n]=x[2 n+1], \\
& x_{e}[n]=x[2 n] .
\end{aligned}
$$

(2) Predict: the prediction operator $P$ is used to predict the odd sample set $X_{o}$ based on the even sample set $X_{e}$. Then, the prediction error between $x_{o}[n]$ and $P\left(X_{e}\right)$ gives the detail coefficients $d[n]$ :

$$
d[n]=x_{o}[n]-P\left(X_{e}\right) .
$$

(3) Update: use the update operator $U$ to update the detail coefficients $D=\{d[n], n \in Z\}$ and add the result $U(D)$ to $x_{e}[n]$; the approximation coefficients $c[n]$ can be obtained:

$$
c[n]=x_{e}[n]+U(D) .
$$

After the above three steps, the detail coefficients $D=\{d[n], n \in Z\}$ and the approximation coefficients $C=\{c[n], n \in Z\}$ are obtained. Multilayer decomposition of SGW can be carried out through the iteration of these three steps. Here, the prediction operator 


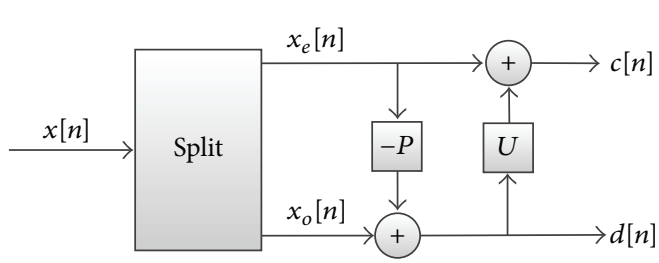

(a)

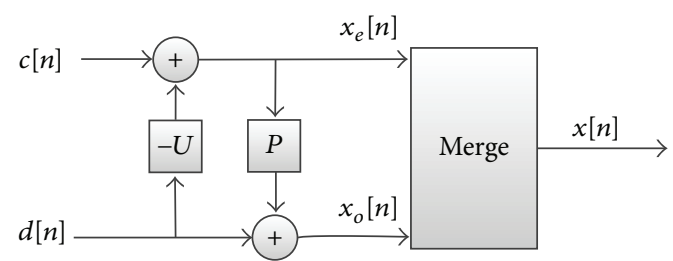

(b)

FIGURE 1: Principle of SGW transform. (a) Decomposition of SGW. (b) Reconstruction of SGW.

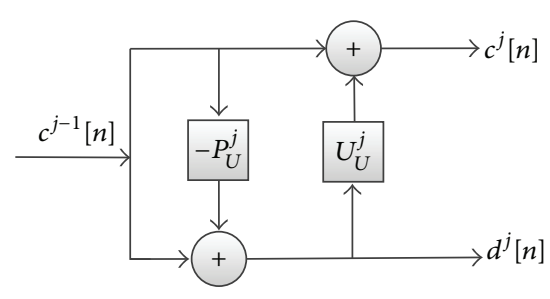

(a)

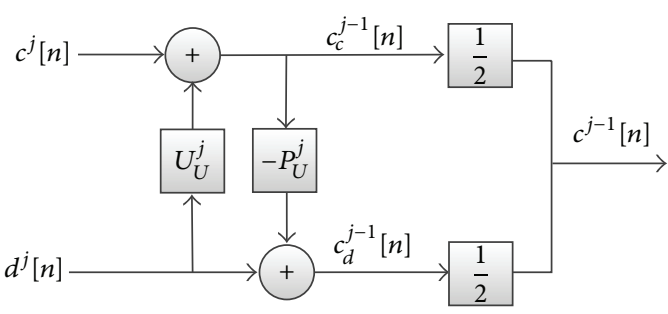

(b)

Figure 2: Principle of RSWG transform. (a) Decomposition of RSWG. (b) Reconstruction of RSWG.

$P=[p(1), p(2), \ldots, p(M)]$ and update operator $U=$ $[u(1), u(2), \ldots, u(N)]$ are vectors with length of $M$ and $N$, respectively. They can be designed by the interpolating subdivision method [29]. The obtained SGW is denoted as $(M, N)$ SGW.

With the decomposition transform of SGW, the reconstruction transform of SGW can be implemented easily by inversing (1), (2), and (3).

2.2. Summary of Redundant Second-Generation Wavelet Theory. Although SGW has got extensive application, it suffers from some defects. Because of the split and merge operations in SGW transformation processes, frequency aliasing problem may be encountered. This will cause wrong estimation of the noise intensity in signal and degrade the denoising result.

RSGW does not need split and merge in its transformation processes and thus can overcome the frequency aliasing problem to a large extent. Assume that $P_{U}^{j}$ and $U_{U}^{j}$ are the prediction operator and the update operator of RSGW at the $2^{j}$ scale, respectively; the initial prediction operator $P_{U}^{0}=$ $\left[p^{0}(1), p^{0}(2), \ldots, p^{0}(M)\right]$ and the initial update operator $U_{U}^{0}=\left[u^{0}(1), u^{0}(2), \ldots, u^{0}(N)\right]$ can be calculated according to the prediction operator length $M$ and the update operator length $N$; then $P_{U}^{j}$ and $U_{U}^{j}$ can be calculated as [27]

$$
\begin{aligned}
P_{U}^{j} & =[p^{0}(1), \underbrace{0, \ldots, 0}_{2^{j}-1}, p^{0}(2), \underbrace{0, \ldots, 0}_{2^{j}-1}, p^{0}(3), \ldots, \\
& p^{0}(M-1), \underbrace{0, \ldots, 0}_{2^{j}-1}, p^{0}(M)],
\end{aligned}
$$

$$
\begin{aligned}
& U_{U}^{j}=[u^{0}(1), \underbrace{0, \ldots, 0}_{2^{j}-1}, u^{0}(2), \underbrace{0, \ldots, 0}_{2^{j}-1}, u^{0}(3), \ldots, \\
& u^{0}(N-1), \underbrace{0, \ldots, 0}_{2^{j}-1}, u^{0}(N)] .
\end{aligned}
$$

The principle of RSGW transform at $2^{j}$ scale is shown in Figure 2. And the decomposition equation of RSGW at $2^{j}$ scale is expressed as

$$
\begin{aligned}
& d^{j}[n]=c^{j-1}[n]-P_{U}^{j}\left(C^{j-1}\right), \\
& c^{j}[n]=c^{j-1}[n]+U_{U}^{j}\left(D^{j}\right) .
\end{aligned}
$$

Correspondingly, the reconstruction equation of RSGW at $2^{j}$ scale is expressed as

$$
\begin{aligned}
& c_{c}^{j-1}[n]=c^{j}[n]-U_{U}^{j}\left(D^{j}\right) \\
& c_{d}^{j-1}[n]=d^{j}[n]+P_{U}^{j}\left(C_{c}^{j-1}\right) \\
& c^{j-1}[n]=\frac{1}{2} c_{c}^{j-1}[n]+\frac{1}{2} c_{d}^{j-1}[n] .
\end{aligned}
$$

\section{Signal Denoising Method Based on Adaptive Redundant Second-Generation Wavelet}

As mentioned above, RSGW is superior to SGW in the aspect of frequency aliasing problem. However, the prediction operator length $M$, update operator length $N$, and decomposition 


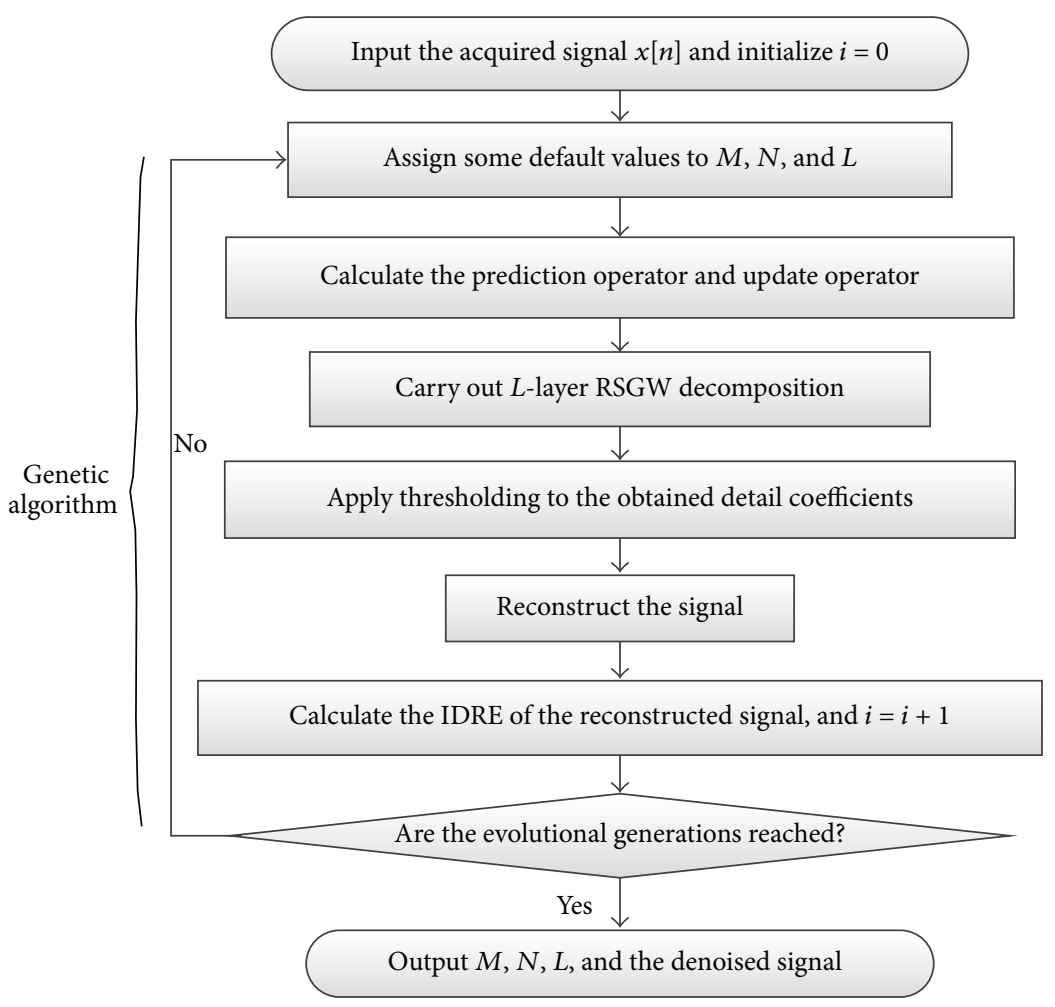

FIGURE 3: Flow chart of ARSGW denoising method.

level $L$ are often predetermined based on experience in the process of vibration signal denoising. Inappropriate selection of these parameters may lead to unsatisfactory denoising result.

To overcome the drawbacks of RSGW, an adaptive redundant second-generation wavelet denoising method is proposed. This section will discuss in detail the new denoising method based on RSGW. To obtain optimal parameters of RSGW, an optimization algorithm is needed. Genetic algorithm can generate near-optimal solutions in complex and nonlinear search spaces with time efficiency [30] and solve large-scale problems [31]. Considerable research has applied genetic algorithm-based methods to machinery fault diagnosis [32-34]. Therefore, in the proposed method, genetic algorithm is taken as the optimization algorithm to search for the optimal parameters of $M, N$, and $L$. Because the parameters $M, N$, and $L$ can change adaptively according to the signal characteristics using ARSGW denoising method, better denoising result can be obtained.

3.1. Procedure of the Denoising Method. The flow chart of the proposed method is shown in Figure 3, and the specific procedure is given as follows:

(1) Input the acquired vibration signal $x[n]$ and initialize the iteration variable $i=0$.

(2) Assign some default values to the adaptive parameters $M, N$, and $L$.

(3) Calculate the prediction operator and update operator according to (4).
(4) According to (5), carry out L-layer RSGW decomposition on the signal $x[n]$.

(5) Apply thresholding to the obtained detail coefficients to suppress the signal noise.

(6) Reconstruct the signal with the processed detail coefficients and the approximation coefficients according to $(6)$.

(7) Calculate the IDRE of the reconstructed signal, and increase the iteration variable $i$ to $i+1$.

(8) Compare the value of the iteration variable with the evolutional generations. Turn to step (2) if the value of the iteration variable is smaller; otherwise output the optimized parameters $M, N, L$, and the denoised signal.

3.2. Index for Denoising Result Evaluation. It can be seen from Figure 3 that, to realize the optimal denoising of rotating machinery vibration signal, an index for signal denoising result evaluation is essential to be the optimization objective. SNR is a ratio between the real signal and the noise component. A larger SNR means that the denoising result is better. However, because the real signal of rotating machinery vibration signal is unknown beforehand, the SNR could not be calculated. Therefore, IDRE is constructed for the rotating machinery vibration signal.

Generally, signal noise is mainly divided into white Gaussian noise, colored noise, and impulsive noise. Among these types, white Gaussian noise, easily aroused by signal 
recording device and transmission system [35], is commonly contained in vibration signal. Numerous methods have been invited into denoising of the signal contaminated with white Gaussian noise, such as EMD [10], wavelet [13], and multiwavelets [14]. The denoising method proposed in this paper is also mainly applied to denoising of white noise.

Assume that the acquired vibration signal $x[n]$ is composed of the real signal $f[n]$ and white Gaussian noise component $\varepsilon[n]$; namely, $x[n]=f[n]+\varepsilon[n]$; then, the autocorrelation function of $x[n]$ can be expressed as

$$
\begin{aligned}
r_{x}[m] & =\frac{1}{N} \sum_{n=1}^{N}(f[n]+\varepsilon[n])(f[n+m]+\varepsilon[n+m]) \\
& =r_{f}[m]+r_{f \varepsilon}[m]+r_{\varepsilon f}[m]+r_{\varepsilon}[m]
\end{aligned}
$$

where $r_{f}[m]$ and $r_{\varepsilon}[m]$ are the autocorrelation functions of $f[n]$ and $\varepsilon[n]$, respectively, and $r_{f_{\varepsilon}}[m]$ and $r_{\varepsilon f}[m]$ are the cross correlation functions of $f[n]$ and $\varepsilon[n]$, respectively.

Analyzing (7), the following can be known:

(1) The noise component $\varepsilon[n]$ is random and irrelevant with the real signal $f[n]$. Hence, $r_{f \varepsilon}[m]$ and $r_{\varepsilon f}[m]$ are almost equal to zero; the maximum value of $r_{\varepsilon}[\mathrm{m}]$ appears at $m=0$ and as $m$ increases, the value of $r_{\varepsilon}[m]$ decreases rapidly.

(2) For a rotating machinery signal, $f[n]$ is mainly composed of period signal components, and so is $r_{f}[\mathrm{~m}]$. In addition, when $f[n]$ contains signal component with the period of $T, r_{f}[m]$ has maximum values at $m=0, T, 2 T, \ldots$.

Considering the above analysis, at the point $m=0, r_{x}[m]$ has a maximum value $r_{x}^{\max 1}$, and the value of $r_{x}^{\max 1}$ is related to the real signal $f[n]$ and the intensity of the noise component $\varepsilon[n]$. When $f[n]$ is fixed, the more intense the noise, the larger $r_{x}^{\max 1}$ will be. Suppose that $r_{x}^{\max 2}$ is the secondary maximum value of $r_{x}[m]$. Since as $m$ increases from 0 , the value of $r_{\varepsilon}[m]$ decreases rapidly, $r_{x}^{\max 2}$ mainly depends on $r_{f}[m]$ and related to the real signal $f[n]$. Hence, when $f[n]$ is fixed, $r_{x}^{\max 2}$ keeps nearly the same. Therefore, IDRE is constructed as

$$
\operatorname{IDRE}=\frac{r_{x}^{\max 2}}{r_{x}^{\max 1}}
$$

It can be concluded from (8) that the fewer the noise components the signal contains, the better the denoising result, and the larger the value of IDRE.

\section{Simulation Signal Denoising}

4.1. Construction of Hydroturbine Unit Vibration Simulation Signal. Assume the rotating frequency $f$ of a hydroturbine unit vibration signal is $1.25 \mathrm{~Hz}$. According to the characteristics of hydroturbine unit vibration signal, a signal function,
TABLE 1: Settings of some relevant parameters used in genetic algorithm.

\begin{tabular}{lc}
\hline Parameters & Values \\
\hline Decomposition layer $L$ & Integer within $[1,6]$ \\
Prediction operator length $M$ & Even number within $[2,14]$ \\
Update operator length $N$ & Even number within $[2,14]$ \\
Population scale & 30 \\
Evolution generation & 20 \\
Probability of crossover & 0.8 \\
Probability of mutation & 0.02 \\
\hline
\end{tabular}

composed of $0.2 \sim 0.45 f, f, 2 f, 3 f$, and $4 f$ signal components, is given as

$$
\begin{aligned}
f(t)= & 20 \sin (2.5 \pi t)+4.5 \sin (5 \pi t)+2.55 \sin (7.5 \pi t) \\
& +1.5 \sin (10 \pi t)+0.4 \sin (0.5 \pi t) \\
& +0.3 \sin (0.75 \pi t)
\end{aligned}
$$

Assuming the sampling frequency is $2048 \mathrm{~Hz}$ and the measurement time is $10 \mathrm{~s}$, the signal without noise can be obtained as shown in Figure 4(a). Adding Gauss white noise $\varepsilon(t)$ with $\mathrm{SNR}_{\mathrm{in}}=18 \mathrm{db}$ to this signal, a noisy vibration signal $x(t)=f(t)+\varepsilon(t)$ can be constructed as shown in Figure 4(b).

\subsection{Hydroturbine Unit Vibration Simulation Signal Denoising.} The ARSGW denoising method is adopted to denoise the constructed hydroturbine unit vibration simulation signal. In this process, settings of some relevant parameters used in genetic algorithm are listed in Table 1.

After performing the procedure illustrated in Section 3.1, the optimal denoising result can be obtained as shown in Figure 4(c). The optimal parameters $M=2, N=2, L=5$, $\mathrm{SNR}_{\text {out }}=27.3924$, and IDRE $=0.9987$.

To illustrate the effectiveness of ARSGW and IDRE on getting the optimal signal denoising result and the denoising result evaluation, respectively, the constructed signal is also denoised by RSGW with different values of $M, N$, and $L$. Table 2 shows $\mathrm{SNR}_{\text {out }}$ and IDRE of the denoised signal with $L$ setting to the integer within $[1,6]$ and $(M, N)$ setting to $(2,2)$, $(2,4),(4,2)$, and $(4,4)$, respectively.

From Table 2, the following conclusions can be reached:

(1) When using RSGW to denoise the constructed signal, the optimal denoising result is obtained when the parameters $M=2, N=2$, and $L=5$. In this case, the obtained $\mathrm{SNR}_{\text {out }}=27.3924$ and $\mathrm{IDRE}=0.9987$. These values are equal to those obtained by ARSGW denoising method, which indicates that the ARSGW denoising method can find out the optimal denoising result exactly.

(2) The variation tendency of IDRE is consistent with that of $\mathrm{SNR}_{\text {out }}$, which indicates the IDRE could be used to evaluate the denoising result.

To test the effectiveness of IDRE further, denoising simulation when there is no noise was conducted. To simulate 


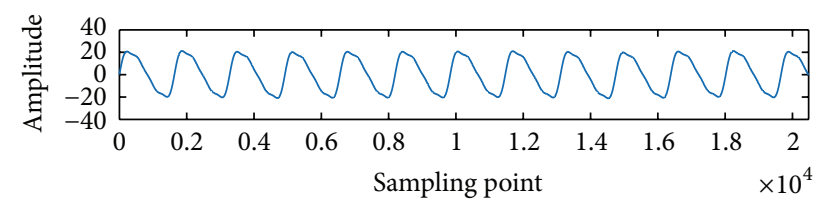

(a)

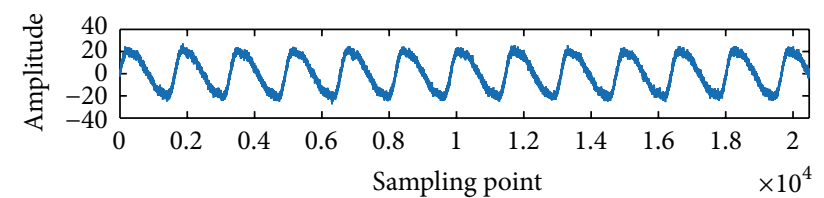

(b)

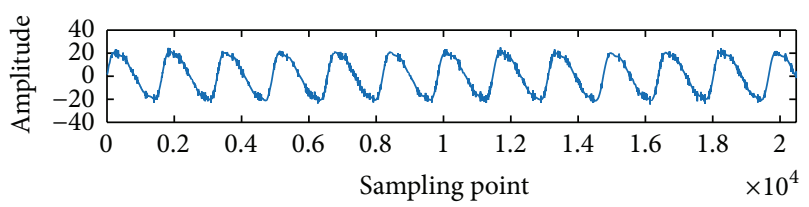

(c)

FIGURE 4: Hydroturbine unit vibration simulation signal denoising based on ARSGW denoising method. (a) Real signal, (b) noisy signal, and (c) denoised signal.

the no-noise condition, $\mathrm{SNR}_{\text {in }}$ was set to 1000 , and other parameters were set as the optimal ones obtained above. The result shows that the value of IDRE equals 1 . This is consistent with (8) that when there is no noise, $r_{x}^{\max 1}$ and $r_{x}^{\max 2}$ are equal.

Compared with SNR, because IDRE can be calculated without needing to know the real signal beforehand, it is suitable not only to simulation signal denoising result evaluation but also to actual vibration signal denoising result evaluation. Therefore, the maximum value of IDRE can be taken as the optimization objective when ARSGW denoising method is adopted for rotating machinery vibration signal denoising.

\section{Vibration Fault Diagnosis for Rotating Machinery}

5.1. Vibration Signal Acquisition. In this paper, the rotating machinery system shown in Figure 5 is used to generate vibration signals under different operating conditions. The rotor with a diameter of $10 \mathrm{~mm}$ and a length of $850 \mathrm{~mm}$ is driven by a DC motor controlled by a speed controller. It is composed of two single shafts coupled together and supported by four bearing blocks. Two mass disks with a diameter of $75 \mathrm{~mm}$ are fixed on the rotor and two rub screw housings are installed on the rack of the system. The sensors for signal acquisition are composed of two eddy current sensors for displacement measurement, a photoelectric sensor for speed measurement, and a piezoelectric accelerometer for vibration measurement. The signals measured by eddy current sensors and piezoelectric accelerometer are sent to a proximitor for filtering and amplification and then transmitted to the computer for storage and analysis.
TABLE 2: Denoising results by RSGW denoising method.

\begin{tabular}{|c|c|c|c|c|}
\hline$L$ & $M$ & $N$ & $\mathrm{SNR}_{\text {out }}$ & IDRE \\
\hline \multirow{4}{*}{1} & 2 & 2 & 21.8930 & 0.9933 \\
\hline & 2 & 4 & 21.7229 & 0.9931 \\
\hline & 4 & 2 & 21.7341 & 0.9931 \\
\hline & 4 & 4 & 21.6737 & 0.9930 \\
\hline \multirow{4}{*}{2} & 2 & 2 & 23.3813 & 0.9965 \\
\hline & 2 & 4 & 22.9608 & 0.9960 \\
\hline & 4 & 2 & 22.8848 & 0.9959 \\
\hline & 4 & 4 & 22.6166 & 0.9956 \\
\hline \multirow{4}{*}{3} & 2 & 2 & 24.8617 & 0.9977 \\
\hline & 2 & 4 & 24.2330 & 0.9972 \\
\hline & 4 & 2 & 24.0670 & 0.9971 \\
\hline & 4 & 4 & 23.6285 & 0.9966 \\
\hline \multirow{4}{*}{4} & 2 & 2 & 26.2169 & 0.9983 \\
\hline & 2 & 4 & 25.3752 & 0.9979 \\
\hline & 4 & 2 & 25.1298 & 0.9977 \\
\hline & 4 & 4 & 24.5158 & 0.9973 \\
\hline \multirow{4}{*}{5} & 2 & 2 & 27.3924 & 0.9987 \\
\hline & 2 & 4 & 26.3497 & 0.9983 \\
\hline & 4 & 2 & 26.0228 & 0.9976 \\
\hline & 4 & 4 & 25.2368 & 0.9971 \\
\hline \multirow{4}{*}{6} & 2 & 2 & 26.7456 & 0.9985 \\
\hline & 2 & 4 & 25.8345 & 0.9981 \\
\hline & 4 & 2 & 25.5388 & 0.9980 \\
\hline & 4 & 4 & 24.7874 & 0.9975 \\
\hline
\end{tabular}

The considered machine states include normal, unbalance, misalignment, and rotor-to-stator rub state. The unbalance state is simulated by screwing a $2 \mathrm{~g}$ mass block into the threaded hole near the edge of mass disk 1, while the misalignment state is simulated by misaligning the coupling of the rotor. In the rotor-to-stator rub case, screw housing 1 is screwed into a rub screw. During the signal acquisition process, the speed of the system is assigned to $1200 \mathrm{rpm}$, and the sampling frequency is $2048 \mathrm{~Hz}$. A set of signals acquired under these four operating states are shown in Figure 6.

5.2. Vibration Signal Denoising. To validate the effectiveness of the proposed denoising method, both ARSGW and SGW denoising method are used to denoise the acquired 40 sets of vibration signals for comparison.

5.2.1. Signal Denoising Using ARSGW Denoising Method. During the process of ARSGW denoising, the decomposition layer is set to an integer within $[1,4]$; the other relevant parameters are set to the values, the same as those illustrated in Table 1. A set of denoised signals by ARSGW denoising method is shown in Figure 7.

5.2.2. Signal Denoising Using SGW Denoising Method. During the process of SGW denoising, the values of the prediction operator length $M$, the update operator length $N$, and the decomposition layer $L$ are set to the same values with those 


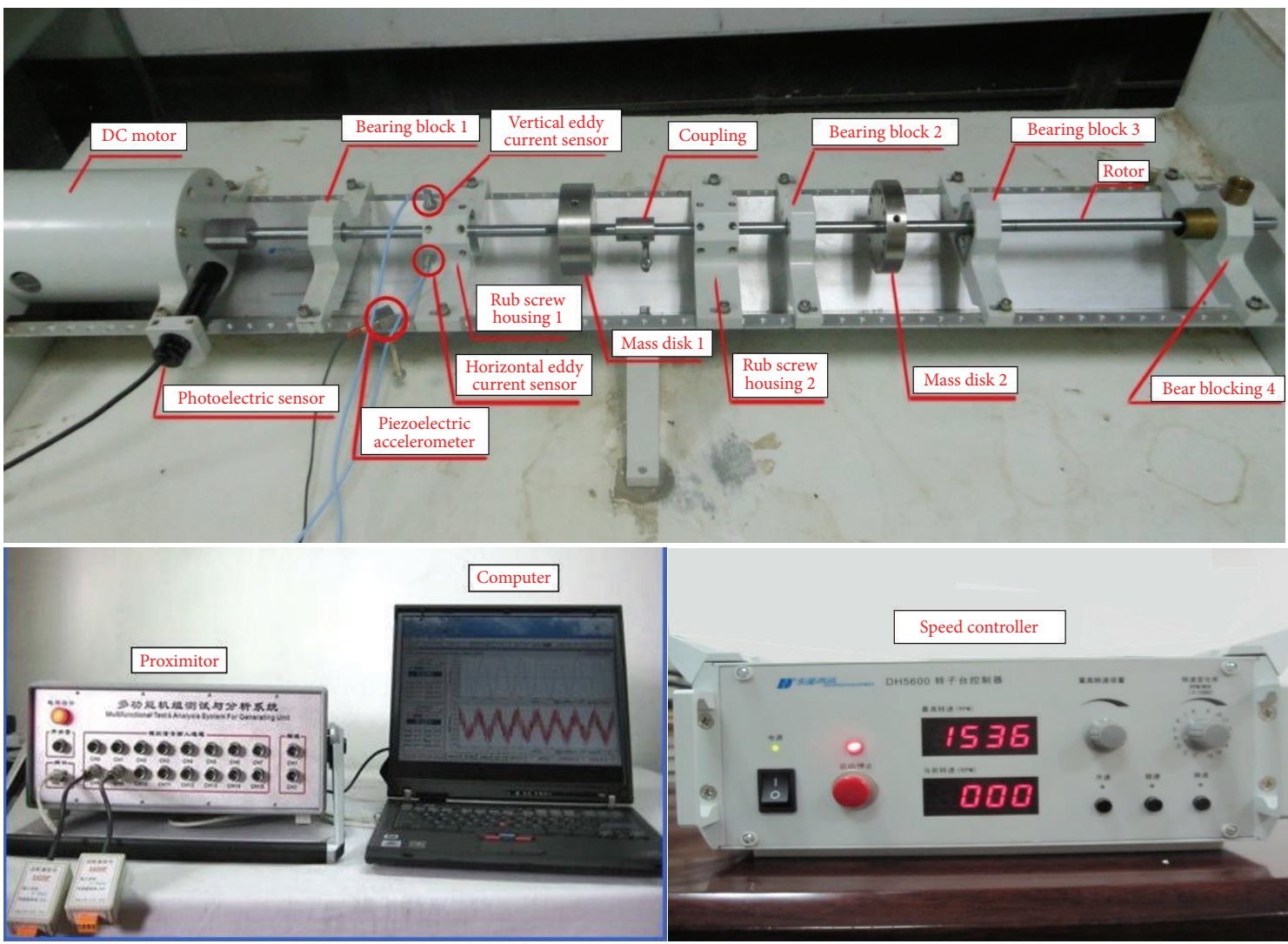

FIGURE 5: The rotating machinery system.
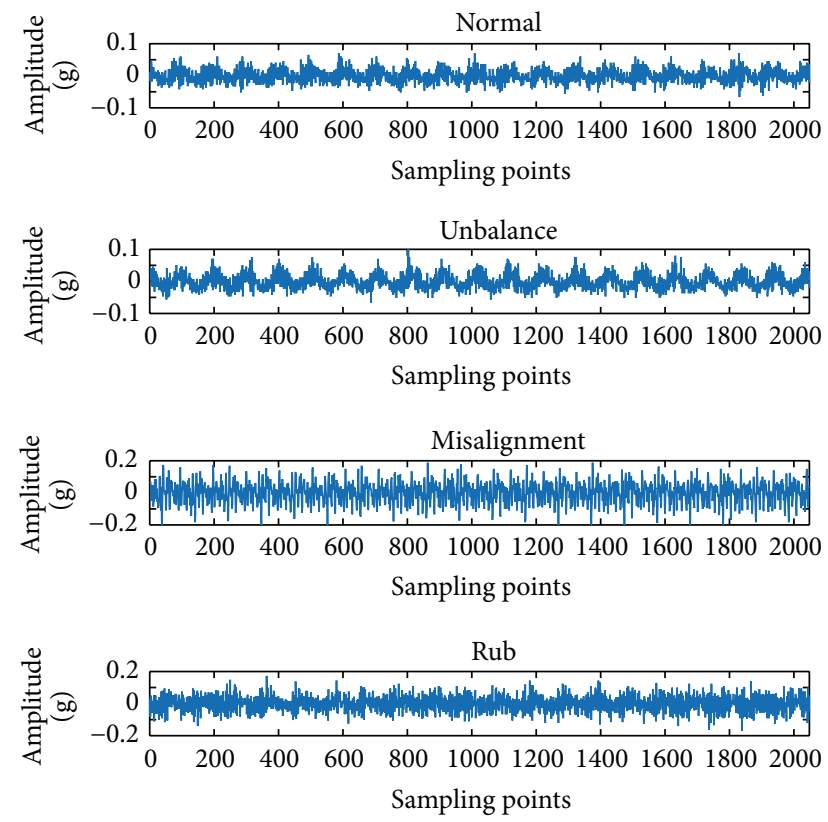

Figure 6: The acquired vibration signals.

assigned in ARSGW denoising method. The waveforms of a set of denoised signals are demonstrated in Figure 8.
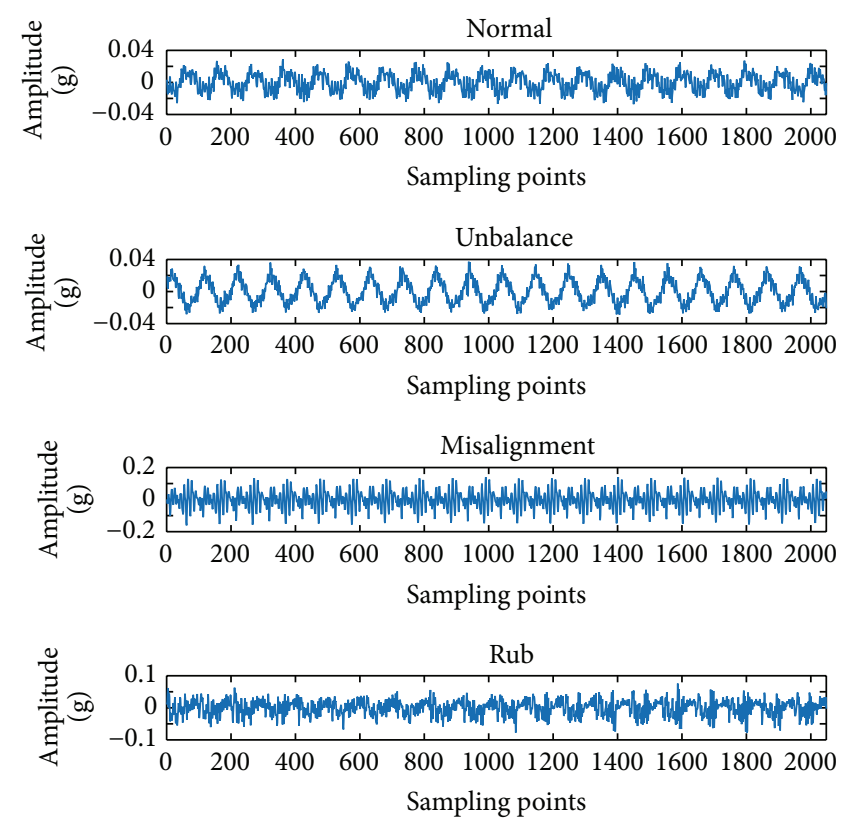

FIGURE 7: Denoising results by ARSGW denoising method.

From the denoised signals shown in Figures 7 and 8, it can be seen that ARSGW denoising method, compared 


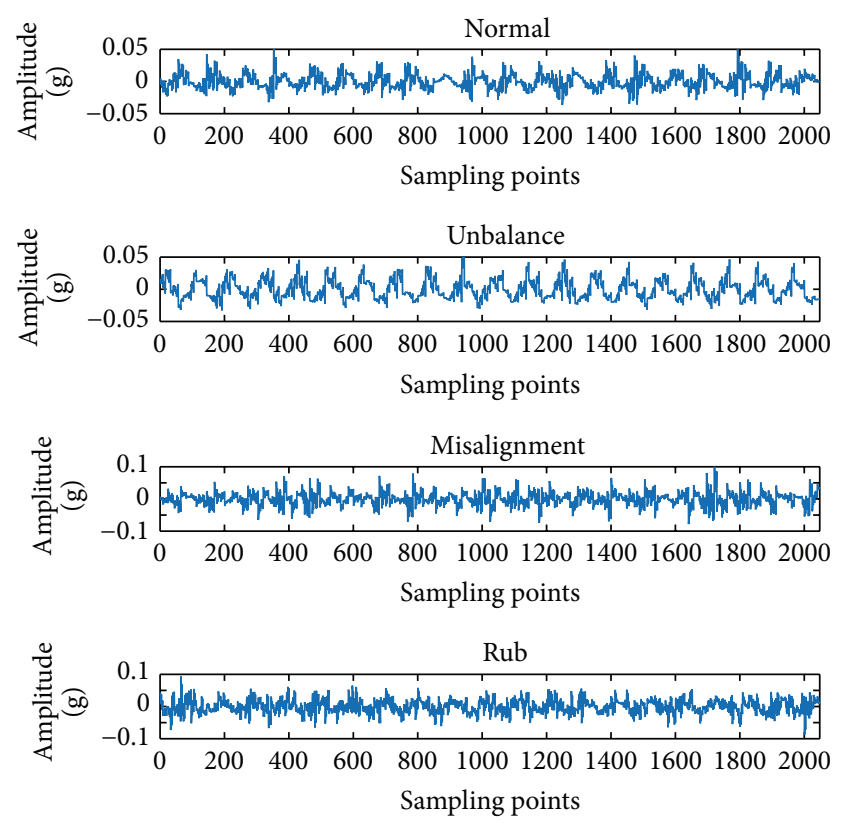

FIGURE 8: Denoising results by SGW denoising method.

TABLE 3: Classification accuracy.

\begin{tabular}{lcccc}
\hline Denoising method & Normal & Unbalance & Misalignment & Rub \\
\hline ARSGW & $100 \%$ & $100 \%$ & $100 \%$ & $90 \%$ \\
SGW & $90 \%$ & 95 & $95 \%$ & $90 \%$ \\
\hline
\end{tabular}

with SWG denoising method, can suppress more noise components and make the periodic features of the signal more obvious; furthermore, the difference among the signals under the four operating states denoised by ARSGW denoising method becomes larger, which is beneficial to the improvement of the fault diagnosis accuracy.

5.3. Rotating Machinery Fault Diagnosis. To further validate the ability of ARSGW denoising method on improving the accuracy of the rotating machinery fault diagnosis, the commonly used support vector machine method [36] is applied for the four operating states' classification.

Firstly, features, including means, variances, $1 f, 2 f$, and $3 f$, are extracted from the 40 sets of signals denoised by ARSGW and SGW denoising method. Then, features of 20 sets of denoised signals are used to train the support vector machine and those of the other 20 sets are used for test. Table 3 illustrates the classification accuracy of the four operating states using ARSGW and SGW denoising method, respectively.

Table 3 shows that the classification result adopting ARSGW as the denoising method is better than that adopting SGW as the denoising method. This indicates that the proposed ARSGW denoising method can effectively improve the accuracy of the rotating machinery fault diagnosis.

\section{Conclusions}

The vibration signal denoising effect directly affects the rotating machinery fault diagnosis performance. In this paper, a new IDRE is constructed, and based on IDRE, ARSGW denoising method is proposed for rotating machinery vibration signal denoising. The application of ARSGW denoising method on the vibration signal denoising of a rotating machinery system gets a better result than that obtained by SGW denoising method. Moreover, after inputting the extracted features from the denoised signal into support vector machine method for classification, the result shows that the recognition rate using ARSGW denoising method is higher than that using SGW denoising method. Therefore, ARSGW denoising method can effectively improve the accuracy of rotating machinery vibration fault diagnosis.

ARSGW denoising method, compared with SGW denoising method, can be applied to denoise rotating machinery vibration signal adaptively and obtain optimal denoising results. The newly constructed IDRE, verified to be effective in evaluating the vibration signal denoising result for rotating machinery, also provides some reference for signal denoising of other kinds of machinery.

\section{Competing Interests}

The authors declare that there is no conflict of interests regarding the publication of this manuscript.

\section{Authors' Contributions}

$\mathrm{Na}$ Lu and Guangtao Zhang contributed equally to this work.

\section{Acknowledgments}

The authors acknowledge the financial support from the National Natural Science Foundation of China under Grant no. 51609203 and from the Fundamental Research Funds for the Central Universities under Grant no. 2014BSJJ053.

\section{References}

[1] C. Zhang, B. Li, B. Chen, H. Cao, Y. Zi, and Z. He, "Weak fault signature extraction of rotating machinery using flexible analytic wavelet transform," Mechanical Systems and Signal Processing, vol. 64-65, pp. 162-187, 2015.

[2] L. Ming, L. Fucai, J. Beibei, B. Huiyu, L. Hongguang, and M. Guang, "Multi-fault diagnosis of rotor system based on differential-based empirical mode decomposition," Journal of Vibration and Control, vol. 21, no. 9, pp. 1821-1837, 2015.

[3] N. Li, J. Yang, R. Zhou, and Q. Wang, "Knock detection in spark ignition engines using a nonlinear wavelet transform of the engine cylinder head vibration signal," Measurement Science and Technology, vol. 25, no. 11, Article ID 115002, 2014.

[4] W. Li, Z. Zhu, F. Jiang, G. Zhou, and G. Chen, "Fault diagnosis of rotating machinery with a novel statistical feature extraction and evaluation method," Mechanical Systems and Signal Processing, vol. 50-51, pp. 414-426, 2015. 
[5] W. Du, Y. Li, J. Yuan, and C. Liu, "Denoising with advanced stepwise false discovery rate control and its application to fault diagnosis," Measurement, vol. 45, no. 6, pp. 1515-1526, 2012.

[6] Y. Wang, Z. He, and Y. Zi, "Enhancement of signal denoising and multiple fault signatures detecting in rotating machinery using dual-tree complex wavelet transform," Mechanical Systems and Signal Processing, vol. 24, no. 1, pp. 119-137, 2010.

[7] W. He, Y. Zi, B. Chen, S. Wang, and Z. He, “Tunable Q-factor wavelet transform denoising with neighboring coefficients and its application to rotating machinery fault diagnosis," Science China Technological Sciences, vol. 56, no. 8, pp. 1956-1965, 2013.

[8] J. Tian, C. Morillo, M. H. Azarian, and M. Pecht, "Motor bearing fault detection using spectral kurtosis-based feature extraction coupled with K-nearest neighbor distance analysis," IEEE Transactions on Industrial Electronics, vol. 63, no. 3, pp. 1793-1803, 2016.

[9] Q. He, X. Wang, and Q. Zhou, "Vibration sensor data denoising using a time-frequency manifold for machinery fault diagnosis," Sensors, vol. 14, no. 1, pp. 382-402, 2013.

[10] Y. Kopsinis and S. McLaughlin, "Development of EMD-based denoising methods inspired by wavelet thresholding," IEEE Transactions on Signal Processing, vol. 57, no. 4, pp. 1351-1362, 2009.

[11] A. Nicknam, M. H. Hosseini, and A. Bagheri, "Damage detection and denoising in two-dimensional structures using curvelet transform by wrapping method," Archive of Applied Mechanics, vol. 81, no. 12, pp. 1915-1924, 2011.

[12] Y. Chen, P. Zhang, Z. Wang, W. Yang, and Y. Yang, "Denoising algorithm for mechanical vibration signal using quantum Hadamard transformation," Measurement, vol. 66, pp. 168-175, 2015.

[13] G. S. Vijay, H. S. Kumar, P. P. Srinivasa, N. S. Sriram, and R. B. K. N. Rao, "Evaluation of effectiveness of wavelet based denoising schemes using ANN and SVM for bearing condition classification," Computational Intelligence and Neuroscience, vol. 2012, Article ID 582453, 12 pages, 2012.

[14] H. Sun, K. Li, H. Wang, P. Chen, and Y. Cao, "Intelligent mechanical fault diagnosis based on multiwavelet adaptive threshold denoising and MPSO," Mathematical Problems in Engineering, vol. 2014, Article ID 142795, 15 pages, 2014.

[15] S. De Ridder, X. Neyt, N. Pattyn, and P.-F. Migeotte, "Comparison between EEMD, wavelet and FIR denoising: influence on event detection in impedance cardiography," in Proceedings of the 33rd Annual International Conference of the IEEE Engineering in Medicine and Biology Society (EMBS '11), pp. 806-809, IEEE, Boston, Mass, USA, September 2011.

[16] J.-L. Lin, J. Y.-C. Liu, C.-W. Li, L.-F. Tsai, and H.-Y. Chung, "Motor shaft misalignment detection using multiscale entropy with wavelet denoising," Expert Systems with Applications, vol. 37, no. 10, pp. 7200-7204, 2010.

[17] X. Chiementin, B. Kilundu, L. Rasolofondraibe, S. Crequy, and B. Pottier, "Performance of wavelet denoising in vibration analysis: highlighting," Journal of Vibration and Control, vol. 18, no. 6, pp. 850-858, 2012.

[18] Y. Chen, Y. Zi, H. Cao, Z. He, and H. Sun, "A data-driven threshold for wavelet sliding window denoising in mechanical fault detection," Science China Technological Sciences, vol. 57, no. 3, pp. 589-597, 2014.

[19] R. R. Coifman and D. L. Donoho, "Translation-invariant denoising," in Wavelets and Statistics, vol. 103 of Lecture Notes in Statistics, pp. 1-26, Springer, New York, NY, USA, 1995.
[20] N. Lu, Z. Xiao, and O. P. Malik, "Feature extraction using adaptive multiwavelets and synthetic detection index for rotor fault diagnosis of rotating machinery," Mechanical Systems and Signal Processing, vol. 52-53, no. 1, pp. 393-415, 2015.

[21] Z. Liu, Y. Mi, and Y. Mao, "An improved real-time denoising method based on lifting wavelet transform," Measurement Science Review, vol. 14, no. 3, pp. 152-159, 2014.

[22] B. Li, P.-L. Zhang, S.-S. Mi, R.-X. Hu, and D.-S. Liu, "An adaptive morphological gradient lifting wavelet for detecting bearing defects," Mechanical Systems and Signal Processing, vol. 29, pp. 415-427, 2012.

[23] B. Li, P.-L. Zhang, Q. Mao, S.-S. Mi, and P.-Y. Liu, "Gear fault detection using adaptive morphological gradient lifting wavelet," Journal of Vibration and Control, vol. 19, no. 11, pp. 1646-1657, 2013.

[24] W. Bao, R. Zhou, J. Yang, D. Yu, and N. Li, "Anti-aliasing lifting scheme for mechanical vibration fault feature extraction," Mechanical Systems and Signal Processing, vol. 23, no. 5, pp. 1458-1473, 2009.

[25] X. Fan, M. Liang, T. H. Yeap, and B. Kind, "A joint wavelet lifting and independent component analysis approach to fault detection of rolling element bearings," Smart Materials and Structures, vol. 16, no. 5, pp. 1973-1987, 2007.

[26] X. Chen, B. Li, Y. He, and Z. He, "Second generation wavelet finite element and rotor cracks quantitative identification method," Chinese Journal of Mechanical Engineering, vol. 23, no. 2, pp. 195-199, 2010.

[27] W. Bao, W. Wang, R. Zhou, N. Li, J. Yang, and D. Yu, "Application of a two-dimensional lifting wavelet transform to rotating mechanical vibration data compression," Proceedings of the Institution of Mechanical Engineers, Part C: Journal of Mechanical Engineering Science, vol. 223, no. 10, pp. 2443-2449, 2009.

[28] M.Žvokelj, S. Zupan, and I. Prebil, "Non-linear multivariate and multiscale monitoring and signal denoising strategy using Kernel Principal Component Analysis combined with Ensemble Empirical Mode Decomposition method," Mechanical Systems and Signal Processing, vol. 25, no. 7, pp. 2631-2653, 2011.

[29] R. L. Claypoole, R. G. Baraniuk, and R. D. Nowak, "Adaptive wavelet transforms via lifting," in Proceedings of the IEEE International Conference on Acoustics, Speech and Signal Processing, vol. 3, pp. 1513-1516, Seattle, Wash, USA, May 1998.

[30] L. Lu, J. Yan, and C. W. de Silva, "Dominant feature selection for the fault diagnosis of rotary machines using modified genetic algorithm and empirical mode decomposition," Journal of Sound and Vibration, vol. 344, pp. 464-483, 2015.

[31] Z. Yangping, Z. Bingquan, and W. DongXin, "Application of genetic algorithms to fault diagnosis in nuclear power plants," Reliability Engineering and System Safety, vol. 67, no. 2, pp. 153160, 2000.

[32] Y. Zhang and R. B. Randall, "Rolling element bearing fault diagnosis based on the combination of genetic algorithms and fast kurtogram," Mechanical Systems and Signal Processing, vol. 23, no. 5, pp. 1509-1517, 2009.

[33] Y. Liu, Y. Li, Y.-J. Cao, and C.-X. Guo, "Forward and backward models for fault diagnosis based on parallel genetic algorithms," Journal of Zhejiang University: Science A, vol. 9, no. 10, pp. 14201425, 2008.

[34] M. Cerrada, R. V. Sánchez, D. Cabrera, G. Zurita, and C. Li, "Multi-stage feature selection by using genetic algorithms for fault diagnosis in gearboxes based on vibration signal," Sensors, vol. 15, no. 9, pp. 23903-23926, 2015. 
[35] K. Zeng, J. Huang, and M. Dong, "White Gaussian noise energy estimation and wavelet multi-threshold de-noising for heart sound signals," Circuits, Systems, \& Signal Processing, vol. 33, no. 9, pp. 2987-3002, 2014.

[36] P. Santos, L. F. Villa, A. Reñones, A. Bustillo, and J. Maudes, "An SVM-based solution for fault detection in wind turbines," Sensors, vol. 15, no. 3, pp. 5627-5648, 2015. 


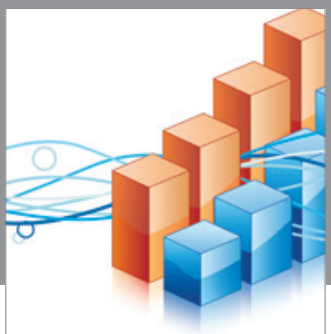

Advances in

Operations Research

vatem alat4

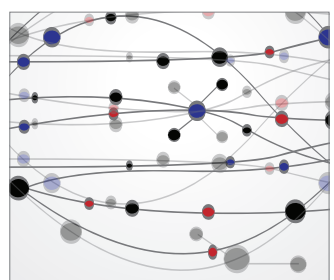

\section{The Scientific} World Journal
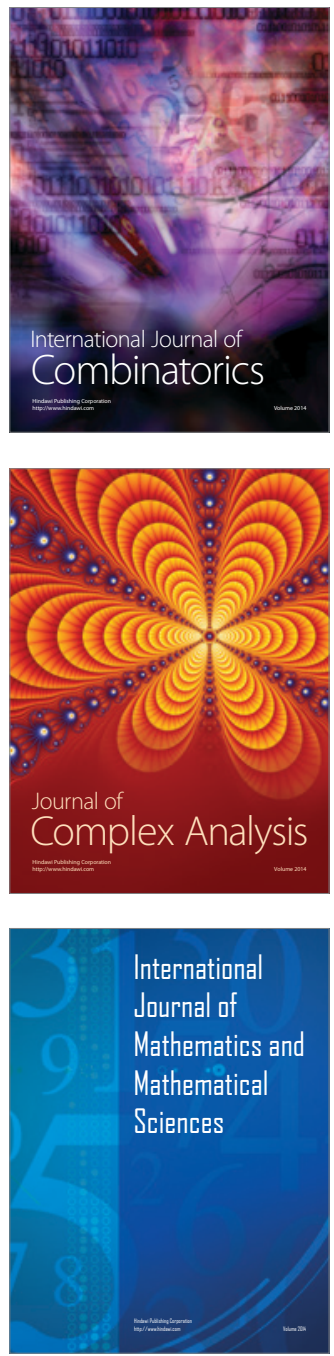
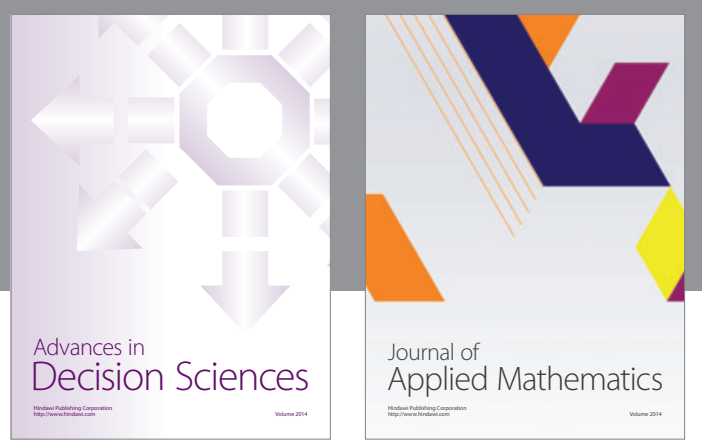

Algebra

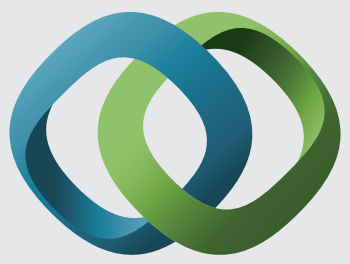

\section{Hindawi}

Submit your manuscripts at

http://www.hindawi.com
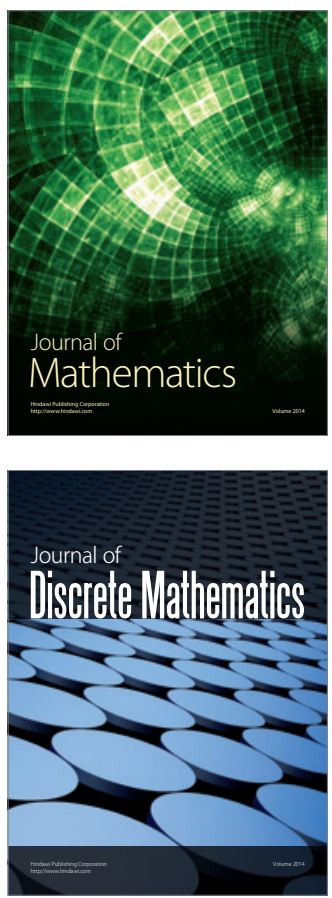

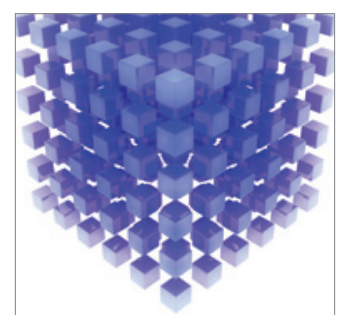

Mathematical Problems in Engineering
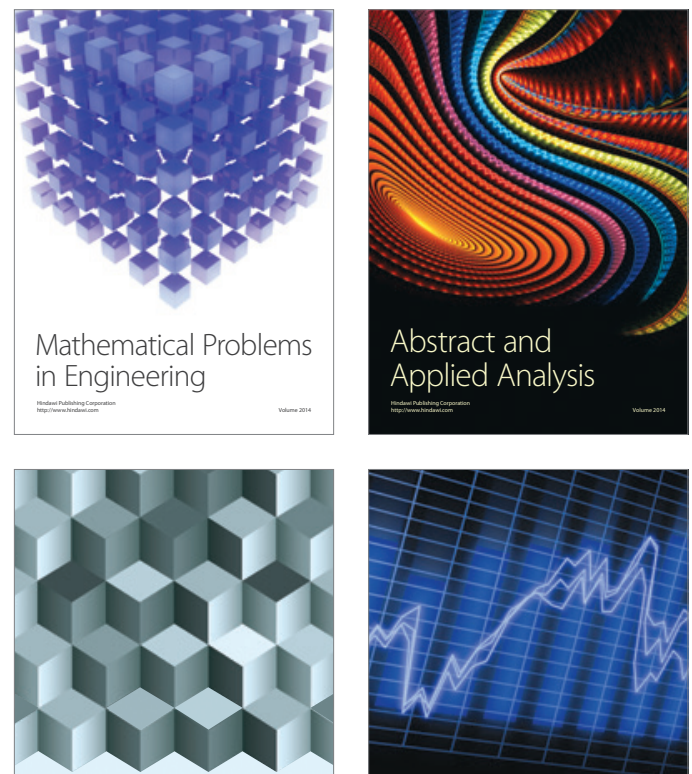

Journal of

Function Spaces

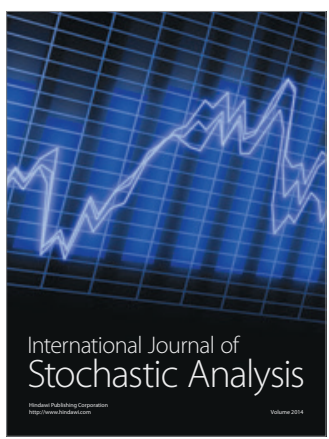

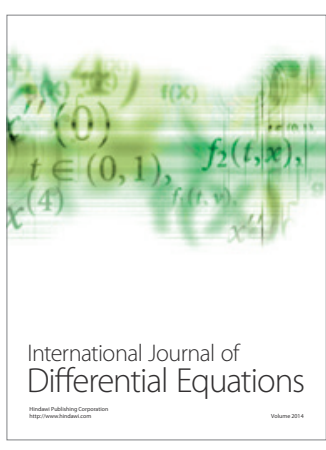
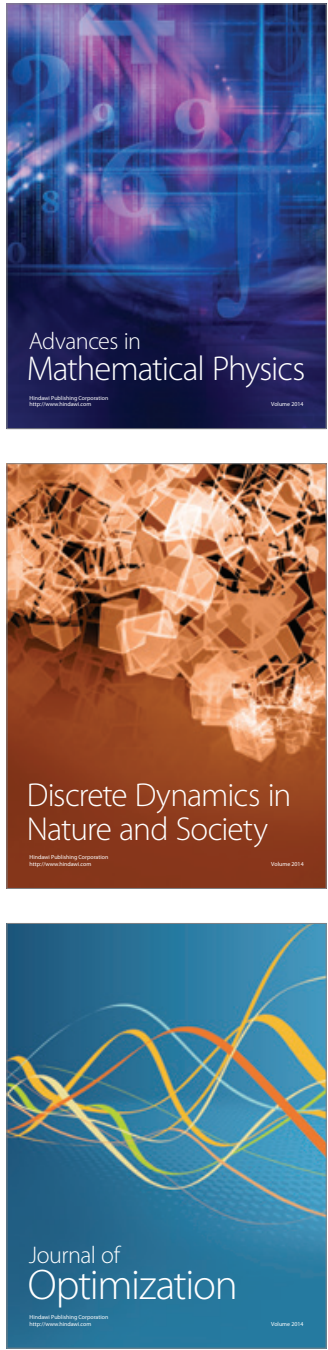